\title{
The attraction of emotions: Irrelevant emotional information modulates motor actions
}

\author{
Elisabetta Ambron • Francesco Foroni
}

Published online: 4 December 2014

(C) Psychonomic Society, Inc. 2014

\begin{abstract}
Emotional expressions are important cues that capture our attention automatically. Although a wide range of work has explored the role and influence of emotions on cognition and behavior, little is known about the way that emotions influence motor actions. Moreover, considering how critical detecting emotional facial expressions in the environment can be, it is important to understand their impact even when they are not directly relevant to the task being performed. Our novel approach was to explore this issue from the attention-and-action perspective, using a task-irrelevant distractor paradigm in which participants are asked to reach for a target while a nontarget stimulus is also presented. We tested whether the movement trajectory would be influenced by irrelevant stimuli-faces with or without emotional expressions. The results showed that reaching paths veered toward faces with emotional expressions, in particular happiness, but not toward neutral expressions. This reinforces the view of emotions as attention-capturing stimuli that are, however, also potential sources of distraction for motor actions.
\end{abstract}

Keywords Reaching movements · Distractor effect .

Emotional faces $\cdot$ Happy face $\cdot$ Angry face

While navigating in daily life, we are bombarded by stimuli of all sorts that may grab our attention. In order to successfully perform in such complex environment, our attentional system needs to sort relevant from irrelevant information. However, irrelevant information may attract our attention exogenously and influence our behavior (Moher \& Song, 2013), without us

E. Ambron $(\triangle) \cdot$ F. Foroni

SISSA-International School for Advanced Studies, via Bonomea,

265, 34136 Trieste, Italy

e-mail: eambron@sissa.it

E. Ambron

e-mail: eli.ambron@gmail.com being fully aware of this process. Contemporarily, the ability to automatically detect emotional expressions, even when we are engaged in another task, is essential for survival, in order to execute the appropriate actions or initiate/avoid important social interactions (cf. Fockenberg, Koole, Lakens, \& Semin, 2013; Hodsoll, Viding, \& Lavie, 2011). Failing to detect an angry expression on the street, for instance, may result in unpleasant detrimental consequences, just as being unable to detect a smiling potential mate across the bar may lead to missing out on an opportunity. In this sense, emotions are important cues that capture our attention automatically (Langton, Law, Burton, \& Schweinberger, 2008) and potentially drive our actions.

The role of emotions extends to all aspects of cognition and behavior (see Cacioppo \& Gardner, 1999), from attention and perception (Niedenthal \& Kitayama, 1994; Zajonc, 1998), to memory (Bradley et al., 1995; Cahill, 1996; Phelps \& Anderson, 1997), reasoning, and decision making (Forgas, 1995; Johnson \& Tversky, 1983; Schwarz \& Clore, 1996). A growing body of research has investigated the effects of emotional facial expressions on attention with respect to neutral faces, by implementing a visual search task and showing consistently that the emotional content of faces affects the extent to which attention is paid to a face, producing a detrimental impact on task performance (Hodsoll et al., 2011).

Although the role of emotions has been widely investigated, little is known about whether emotions may affect simple reaching movements and interfere with ongoing goal-directed actions. The relevance of this topic is that simple reaching movements allow for the systematic investigation of automatic default motor mechanisms of types that are ubiquitous in our daily lives (see Milner, 1996). Moreover, such research allows for studying the relationship between attention and action, providing a wide range of information, which goes beyond button pressing and reaction times, which are considered a measure of processing and movement planning 
(Rosenbaum, 1985). For instance, the spatial and temporal parameters of reaching movements provide a large variety of additional information, including details regarding movement trajectories that have more direct links to more complex behaviors.

Therefore, the lack of investigation on the distracting role of emotions in reaching movements is somewhat surprising, considering the potential downstream implications for social interaction and for our daily lives. One notable exception in the literature is the work on approach/avoidance tendencies related to emotional stimuli (e.g., Chen \& Bargh, 1999; Eder \& Rothermund, 2008). However, this research traditionally entails the study of individuals' behavioral tendencies toward different classes of target stimuli (e.g., positive or negative pictures), which participants are asked to attend to and act upon explicitly. A large number of studies implementing subliminal or suboptimal stimulus presentation have provided important evidence of the power of emotion. The influence of emotions goes beyond explicit processing and recognition, since emotions can influence our behavior even at a more automatic level (Berridge \& Winkielman, 2003; Foroni \& Semin, 2009, 2011), involving primitive brain structures such as the amygdala (Brooks et al., 2012). Therefore, it would be worth investigating the automatic and implicit effects of irrelevant emotional expressions on simple motor actions.

The tied link between attention and action is well exemplified in the phenomenon known as the distractor effect (Howard \& Tipper, 1997; Welsh \& Elliot, 2004). This describes changes in the spatial and/or temporal aspects of goal-directed reaching movements toward a target when a visual distractor is presented. The presence of this effect suggests that motor responses are planned not only toward the target, but also toward irrelevant stimuli. The successful completion of the reaching movement demonstrates that the response toward the distractor has subsequently been inhibited in order to complete the reaching of the target (Howard \& Tipper, 1997; Welsh \& Elliot, 2004). The degrees of activation and inhibition of the response to the distractor determine the final movement trajectory, which may deviate from the "perfect" reaching path by veering toward to or away from the distractor.

The final movement trajectory is determined by the characteristics of the stimuli, since task-salient distractors are more difficult to suppress, and by participants' ability to inhibit the response toward these nontarget stimuli (Tipper, Howard, \& Houghton, 1998). In support of this view, previous research has reported such alterations for populations with low cognitive resources. Patients with dementia or frontal lobe damage show strong distractor interference in reaching tasks (Aron, Sahakian, \& Robbins, 2003; Simone \& Baylis, 1997), and elderly are impaired in performing goal-directed movements (Carmeli et al., 2003). Young adults, on the other hand, show a less-consistent pattern of motor distractibility: they seem to be able to inhibit the tendency to veer toward a distractor particularly when it is task-irrelevant (Ambron, Della Sala, \& McIntosh, 2012; Welsh \& Elliott, 2005). Notably, when attention and cognitive abilities are reduced as a consequence of stress, distractor interference is enhanced (Sato, Takenaka, \& Kawahara, 2012). A recent study (Ambron et al., 2012) supported the link between reduced cognitive abilities and the distractor effect, showing that the movement trajectories of preschool children, a population with low levels of cognitive resources, dramatically veer toward both task-relevant and task-irrelevant distractors. In the same paradigm, the authors showed that young adults are able to inhibit the interference of task-irrelevant distractors, demonstrating no modification of movement trajectories (see also Song \& Nakayama, 2009). Since Ambron et al.'s experiments were designed to elicit a distractor effect in children by implementing cartoon figures as distractors, the lack of an effect in young adults could have been due to the nature of the distractors rather than to the participants' overall inhibitory capacities. It is plausible that these distractors were simply not salient for young adults.

To date, research in this domain has focused almost exclusively on the investigation of motor distractibility using abstract perceptual visual cues that have had low relevance for young individuals outside the experimental settings (Moher \& Song, 2013; Song \& Nakayama, 2006). Due to the possible detrimental downstream implications of motor distractibility for our daily activities, it is important to explore the boundary conditions of the human capacity for inhibiting the impact of a distractor that is irrelevant for the ongoing action but potentially relevant for the individual.

In the present study, we implemented as task-irrelevant distractors natural-life stimuli-faces with emotional expressions - to explore whether a deviation of the movement path would be automatically elicited in young adults when they were facing stimuli of evolutionary significance (Öhman et al. 2001). It is possible that evolution has sculpted attentional systems to provide preferential access to stimuli with adaptive significance for organisms, such as conspecifics' emotional responses (Spoor \& Kelly, 2004), which may provide information that is critical for survival and social interaction (Darwin, 1872/1904; Öhman, 1993).

We predicted that, as compared to neutral stimuli, emotional expressions would be more attention-grabbing and, thus, distracting. The ubiquity of emotions and their role and influence have been richly documented in previous years (Cacioppo \& Gardner, 1999). However, to our knowledge this study represents the first attempt at exploring the possible biasing effects of emotion information on motor actions. The trajectory of a simple reaching movement should be affected by the presentation of faces with emotional expressions (happiness and anger) as task-irrelevant stimuli, in comparison to faces with neutral expressions (masked or not with visual noise). The more distracting the task-irrelevant stimulus, the larger should be the bias in the reaching trajectories. Although 
people's ability to detect emotional expressions that are irrelevant to their ongoing task could be critical for survival, this feature can also result in a biasing factor that may affect their "motor actions."

\section{Materials and methods}

Twenty-seven right-handed students (14 males, 13 females; mean age $=22.4$ years) performed, on a digital laptop tablet (display area of $260 \times 163 \mathrm{~mm}$ ), a reaching task like the one used by Ambron and collegues (2012), presented using the KiniLab software (Culmer, Levesley, Mon-Williams, \& Williams, 2009). That is, participants were asked to trace a path from a starting point (SP) to a target using a stylus (Fig. 1, left panel).

Each trial started with the stylus placed on the SP (a yellow dot, size: $10 \times 10 \mathrm{~mm}$ ). Five-hundred milliseconds after the start of a trial, the target (a yellow dot, size: $10 \times 10 \mathrm{~mm}$ ) appeared in one of two locations on the top half of the screen (right or left of the center of the screen). Along with the target, a task-irrelevant distractor (a human face, size: $30 \times 30 \mathrm{~mm}$ ), appeared on the left or the right side of the target. These stimuli were arranged symmetrically around the screen midline, $40 \mathrm{~mm}$ apart. Participants were required to reach for the target as quickly as they could and to ignore the distractor. These stimuli were arranged symmetrically around the screen midline.

For each target location, we used two male and two female actors displaying different emotions, obtained from a validated emotional faces database (Olszanowski, Pochwatko, Kukliński, Ścibor-Rylski, \& Ohme, 2008). The pictures portrayed different expressions pertaining to four experimental conditions: (a) a neutral expression; (b) a neutral expression altered with graphic noise, making the expression indistinguishable (i.e., a noisy-neutral expression); (c) an angry expression; and (d) a happy expression (see Fig. 1, right panel). These two emotions were chosen in order to have one with positive and one with negative valence, as it was done in previous research using visual search tasks to explore selective attention (e.g., Fox, Russo, \& Dutton, 2002) and basic attention-capturing mechanisms (Horstmann, Borgstedt, \& Heumann, 2006).

Each picture was presented multiple times in both possible target/distractor combinations (e.g., distractor right of target or distractor left of the target), for a total of 128 trials presented in a random order.

The data reduction followed that done by Ambron et al. (2012). Namely, for each movement trajectory, the mean deviation from the ideal straight-line path joining the SP and target was calculated and collapsed across target positions. Uncompleted movements, which did not reach the target, were regarded as errors and deleted (in total, $2 \%$ of the trials were discarded). Then, an index of motor distractibility was computed by recoding the mean trajectory deviations toward (positive values) or away from (negative values) the distractor across locations, obtaining four indexes (one for each distractor type). A significant deviation from zero would confirm a distractor effect with positive (or negative) deviation indicating the tendency to veer toward (or away from) the distractor during the reaching movement. In this way, it was possible to access the relative deviation of the trajectory, irrespective of distractor location (left vs. right) and whether the deviation was toward or away from the distractor, without the need to add baseline trials without a distractor (cf. Ambron et al., 2012).

Furthermore, two temporal parameters of the movementthe time interval between the stylus contact with the SP and

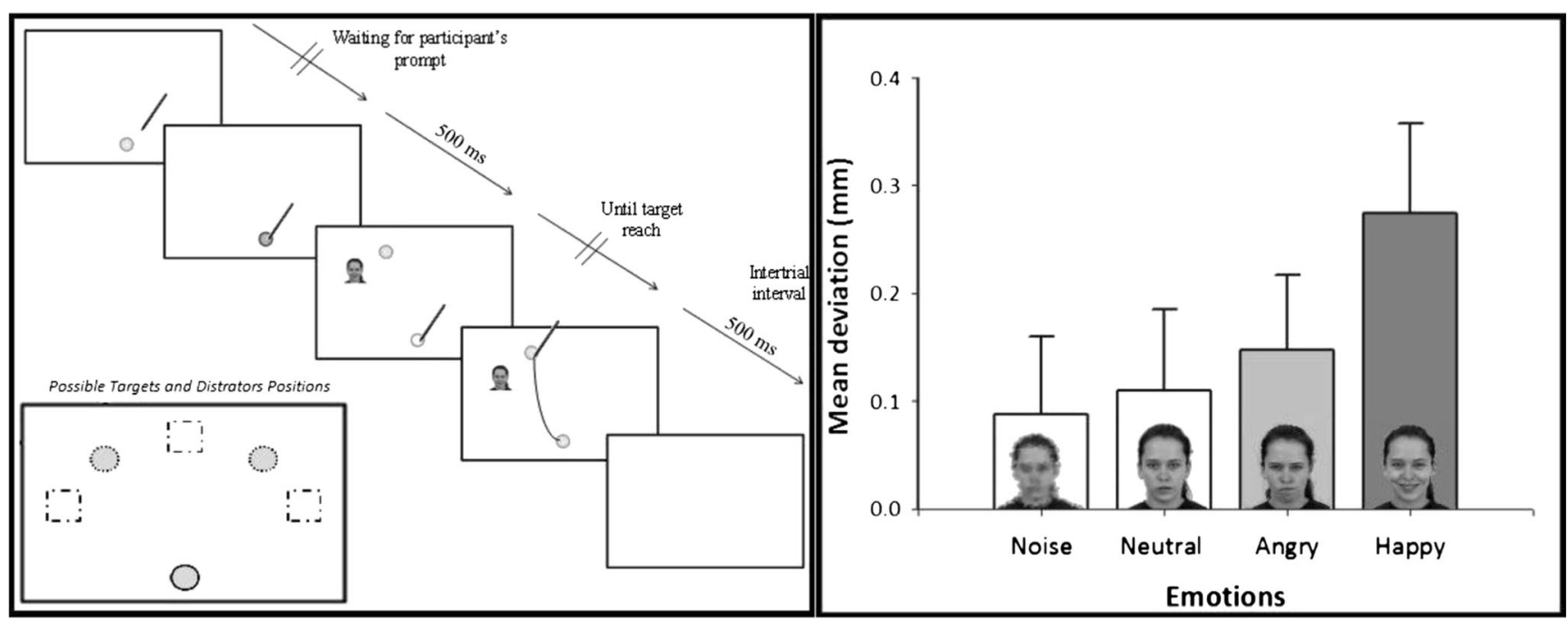

Fig. 1 (Left) Representation of the series of events of a single trial, as well as an illustration of the possible target (dotted circles) and distractor (dotted squares) locations. (Right) Mean deviations toward (positive values) and away from (negative values) the distractor location for the different emotion conditions 
the movement onset (reaction time: RT) and the time between the reaching movement onset and offset (movement time: MT) - were also calculated. They $p$ values were corrected when necessary using Bonferroni correction.

\section{Results}

We first tested for any distractor effect for each distractor type by carrying out one-sample $t$ tests on the motor-distractibility indexes. The analysis confirmed that participants' reaching paths veered significantly toward the distractor when this stimulus displayed emotional content [happy faces, $t(26)$ $=3.3, p<.005$; angry faces, $t(26)=2.1, p<.05]$, whereas the paths did not do so when the distractor displayed neutral expressions [noisy-neutral expression, $t(26)=0.22$, n.s.; neutral expression, $t(26)=0.15$, n.s.]. The noisy-neutral and neutral-expression conditions were not different $[t(26)$ $=0.25$, n.s.], so they were collapsed into a combined control condition.

We then analyzed the effects of the different variables on the motor-distractibility indexes. A mixed analysis of variance, with gender of the participants as a between-subjects factor and emotion (combined control vs. angry vs. happy) and gender of the actor as within-subjects factors, was carried out on the motor-distractibility indexes. Gender was added as factor in the analysis in order to explore whether the distractor effect was due to the gender of the actor in relation to the gender of the participants, to the combination of both the gender and emotion of the face, or to the emotion itself. The results indicated that the gender of the actor or the participant was not important, but that the emotion itself was what mattered, since the only significant result was the main effect of the emotion of the distractor, $F(2,25)=3.15, p=.05, \eta^{2}$ $=11$. This result was driven by a significant difference between the happy and combined-control conditions, $F(2,25)$ $=6.99, p<.05, \eta^{2}=.21$. No other comparison reached significance.

Finally, we analyzed the temporal parameters of the movement using the same mixed analysis of variance design as above. The analyses conducted on the temporal parameters (see Table 1 for the means and $S D$ s) showed only a main effect of the actor's gender on RTs, $F(2,25)=6.4, p<.05, \eta^{2}=.20$, such that participants reacted faster when the actor was a female $(M=869.9, S E=11.7)$ rather than a male $(M=882.1$, $S E=11.9)$.

\section{Discussion}

We tested whether task-irrelevant faces with or without emotional expressions would influence the trajectories of simple reaching movements, under the hypothesis that only faces displaying emotional expressions would affect motor actions. The results supported this hypothesis; trajectories were influenced by and veered toward task-irrelevant faces with emotional expressions. Notably, the movement trajectories were not influenced by neutral expressions of comparable perceptual salience. This finding is in line with the contention that the perceptual and attentional systems may provide preferential access to classes of stimuli with high significance for organisms (Öhman et al. 2001), such as conspecifics' emotional responses (Spoor \& Kelly, 2004). The lack of a distractor effect for neutral faces altered with visual noise suggests that the distracting effect of emotion cannot be due to the complexity of disambiguating an emotional relative to a neutral expression, since the noisy-neutral faces were more difficult to distinguish than the others.

The present results document, for the first time, motor distractibility in reaching movements induced by taskirrelevant distractors represented by conspecifics' emotional expressions. Conspecifics' emotional responses-happiness, in particular (Becker et al., 2012; Spoor \& Kelly, 2004)-are powerful cues that are quickly processed, even if they are presented as distractors (Hodsoll et al., 2011). Indeed, by measuring movement trajectories, we were able to determine that the presence of an emotion induces participants to move toward instead of away from a distractor. We could speculate that the deviation of the reaching path in this sense could be used as an indirect measure of approach and avoidance tendencies, providing additional qualitative implications (Chen \& Bargh, 1999; Eder \& Rothermund, 2008). Since this was not the primary aim of the present study, emotions were not chosen on the basis of their abilities to elicit approach/ avoidance tendencies, but rather for their attention-capturing features (Fox et al., 2002; Horstmann et al., 2006). Future studies will be dedicated to explore this issue specifically using other emotions (fear, disgust, or sadness) or classical stimuli from approach/avoidance paradigms (e.g., flowers/ snakes) as irrelevant distractors. ${ }^{1}$

The result that both happy and angry expressions "attract" movement trajectories substantiates an irrelevant emotionalcapture effect (Hodsoll et al., 2011) and help clarify the potential asymmetry between negative and positive emotional valences (Nasrallah, Carmel, \& Lavie, 2009). Previous research has documented an advantage in detecting negative and threatening emotional facial expressions (Calvo, Avero, \& Lundqvist, 2006; Eastwood, Smilek, \& Merikle, 2001). However, Hodsoll and colleagues have already shown that both angry and happy irrelevant emotional expressions can capture our attention. Similarly, our study has shown that angry and, even more so, happy irrelevant emotional expressions impact movement trajectories, suggesting that this may represent a general effect of emotion. Thus, emotions are able to

\footnotetext{
${ }^{1}$ Thanks to the contribution of an anonymous reviewer.
} 
Table 1 Means and SDs (in milliseconds) of the temporal parameters of the movements

Combined Control (Noisy-Neutral \& Neutral) Emotions

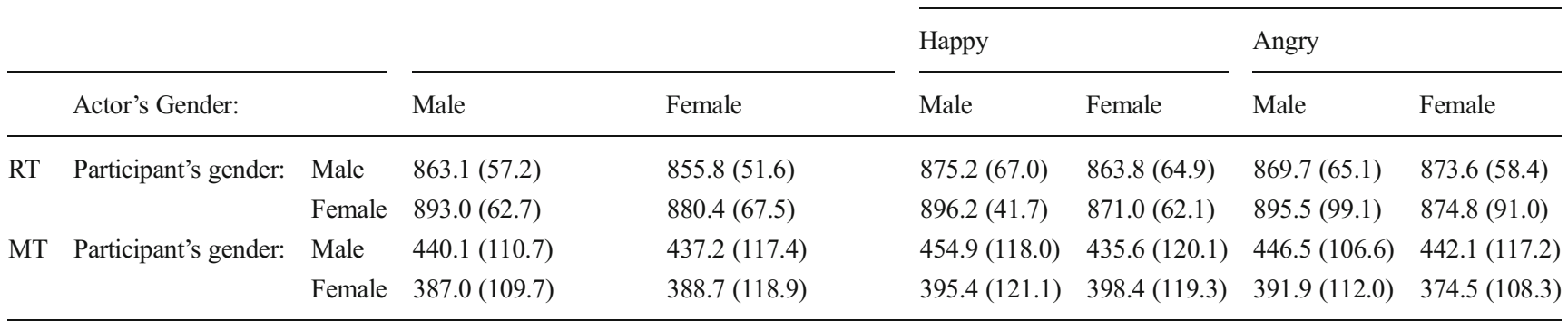

Reaction time (RT) is the interval between the stylus contact with the starting point and the movement onset (including a fixed $500 \mathrm{~ms}$ between stylus contact and the appearance of the target-distractor pair). Movement time (MT) is the time between the reaching movement onset and offset.

automatically capture attention, even if they are irrelevant (Fockenberg et al., 2013; Gainotti, 2012; Hodsoll et al., 2011), and more importantly are able to bias our motor actions.

It is worth emphasizing that the present reaching task was quite simple, and thus an irrelevant nontarget stimulus should have been easy to ignore. Although the target and distractor locations were unpredictable, participants may have realized after a few trials that the target could appear at two possible locations and that a nontarget stimulus would be presented on either side of the target. Moreover, the faces never appeared at the target locations, whereas in other paradigms, such as visual search paradigms, participants are asked to process the stimuli in order to distinguish them from the target. The appearance of a significant effect of emotional expressions on the movement trajectory in this context is, therefore, even more striking. Distraction may be much larger when distractors are unexpected and in unpredictable locations, as occurs in daily-life situations.

We observed no modulations of the movement trajectories by the gender of the actors in relation to the gender of the participants, suggesting that the salience of the emotion occurs regardless of possible mate selection goals (Buss, 1985). Of course, one could argue that the emotional expressions displayed by same- or different-gender actors were salient for the participants on two distinct levels (mate selection and mate competition, respectively). However, the most parsimonious explanation seems to be that conspecifics' emotional responses are in general particularly salient and are not inhibited. Interestingly, the gender of the actors did have an effect on the temporal parameters of the movements, with RTs being faster in trials with female actors as distractors than in trials with male actors. Further research should investigate this issue more in depth.

Within the attention-and-action literature, the present results support the view that salient distractors are more difficult to inhibit (Welsh \& Elliott, 2004, 2005), but we can further specify this account. Rather than the general perceptual characteristics of the distractor, here the "affective" relevance of the distractor emerged as a potential factor modulating motor distractibility. The overall perceptual salience of the face by itself was not sufficient to induce a distractor effect on the movement trajectories, as was shown by the lack of a significant distractor effect in the control conditions. The effect emerged only with stimuli of high significance for young adults, such as peer's emotional expressions as it has been suggested by some authors (Spoor \& Kelly, 2004). This evidence provides an important contribution to this literature, since it specifies further the interplay between attention and motor action. Furthermore, it highlights the role of the subjective relevance of a stimulus in motor distractibility, adding to the role of the perceptual salience of the stimulus itself that has been reported in the literature. Our approach also opens new avenues in the investigation of motor distractibility and its potential moderating factors in relation to special populations. Notably, populations with low cognitive resources, which are thus more prone to the distractor interference, could be more at risk for the downstream implications of the influence of emotions, whereas populations with deficits in emotional processing may be spared by such interference.

The emergence of the distractor effect only in the presence of emotional expressions extends the biasing role of emotions in cognition and behavior also to basic motor actions (Cacioppo \& Gardner, 1999), filling a gap in the literature. The distracting role of emotions translates into being "captured and attracted toward" someone displaying emotional expressions, even when the expression is not relevant to our goal-directed movements. The results are less pronounced for angry than for happy expressions, apparently contradicting research on orienting responses, which has usually also found orienting responses to neutral stimuli (Dimberg \& Öhman 1996; Öhman 1993). However, the research on orienting responses has usually implemented the classical conditioning paradigm, and this may explain the difference.

The result that trajectories veer toward both angry and happy expressions - instead of veering toward happy expressions and away from angry expressions - may seem at variance with what would be predicted by the literature on 
approach/avoidance tendencies (e.g., Chen \& Bargh, 1999; Eder \& Rothermund, 2008). However, it is important to note that participants' goal here was to reach for a target and to ignore the task-irrelevant distractor, and that participants were not requested to attend and process the nontarget stimulus in order to perform the task. Therefore, the present results reflect the automatic and implicit effect of irrelevant emotional expressions on simple motor actions, rather than individuals' behavioral tendency toward attended target stimuli. The present study, on the one hand, highlights the role of emotions as attention-grabbing, and consequently as a potential distracting factor, and on the other hand, it further emphasizes the role of emotions in attention and action tout court. As we mentioned in the introduction, since stress has an impact on the temporal parameters of distractor interference (Sato et al., 2012), future research could investigate this topic by focusing on movement trajectories in particular.

It has been argued that humans have been evolutionarily tuned to respond automatically to facial stimuli. Considering the work showing that simple exposure to facial expressions, even subliminally, impacts subsequent judgments (e.g., Foroni \& Semin, 2009, 2011, 2012), future research should also investigate how the peripheral presentation of taskirrelevant emotional expressions may carry on and, possibly, impact subsequent judgments even of unrelated stimuli.

In conclusion, the present study builds a bridge between the attention-and-action literature and the research on emotion processing, opening the way to new areas of investigation. For instance, future studies should further investigate this distractor effect toward emotional faces that are different in nature and valence (e.g., sad, angry, fearful, happy, and surprise). Moreover, the nature of the stimuli able to elicit this effect could also be investigated. Studies could test whether this effect can be elicited only by real images of faces or can also be observed with schematic cartoon faces and/or emoticons. Other factors should also be investigated, such as the familiarity of the face (e.g., faces of celebrities vs. unknown people). Extrapolating to everyday contexts, the present findings may reflect the tendency for our actions to be influenced by the appearance or presence in the environment of emotional expressions, even when they are irrelevant for the ongoing action. The possible detrimental downstream implications for daily motor activities (e.g., driving) of motor distractibility that is induced by irrelevant emotional expressions in the environment makes it important to further explore the boundary conditions of the human capacity of inhibiting the impact of distractors. For instance, although it is known that the emotional state of a person can influence his or her ability to perform complex action such as driving (Stephens, Trawley, Madigan, \& Groeger, 2013), we can speculate that emotions as external cues, as displayed by a pedestrian or an advertisement, may have similar detrimental effects.
Author note We thank Antimo Buonocore and Andrea Carnaghi for their helpful comments to an early draft of the manuscript, and Laura Facchin for help with the data collection. We also thank Robert D. McIntosh for providing us with custom programs for the kinematic analysis. The writing of this article was supported by a FoodCast Grant (Regione Lombardia) funded by Caritro-Fondazione Cassa di Risparmio di Trento e Rovereto.

\section{References}

Ambron, E., Della Sala, S., \& McIntosh, R. D. (2012). Closing-in behaviour and motor distractibility in children. Neuropsychologia, 50, 419-425.

Aron, A. R., Sahakian, B. J., \& Robbins, T. W. (2003). Distractibility during selection-for-action: Differential deficits in Huntington's disease and following frontal lobe damage. Neuropsychologia, 41, $1137-1147$.

Becker, D. V., Neel, R., Srinivasan, N., Neufeld, S., Kumar, D., \& Fouse, S. (2012). The vividness of happiness in dynamic facial displays of emotion. PLoS ONE, 7, e26551. doi:10.1371/journal.pone.0026551

Berridge, K. C., \& Winkielman, P. (2003). What is an unconscious emotion? (The case for unconscious "liking"). Cognition and Emotion, 17, 181-211.

Bradley, B. P., Mogg, K., \& Williams, R. (1995). Implicit and explicit memory for emotion-congruent information in clinical depression and anxiety. Behaviour research and therapy, 33(7), 755-770.

Brooks, S. J., Savov, V., Allzén, E., Benedict, C., Fredriksson, R., \& Schiöth, H. B. (2012). Exposure to subliminal arousing stimuli induces robust activation in the amygdala, hippocampus, anterior cingulate, insular cortex and primary visual cortex: A systematic meta-analysis of fMRI studies. NeuroImage, 59, 2962-2973. doi:10. 1016/j.neuroimage.2011.09.077

Buss, D. M. (1985). Human mate selection. American Scientist, 73, 4751.

Cacioppo, J. T., \& Gardner, W. L. (1999). Emotion. Annual Review of Psychology, 50, 191-214.

Cahill, L. (1996). Neurobiology of memory for emotional events: converging evidence from infra-human and human studies. Cold Spring Harbor Symposia on Quantitative Biology, 61, 259-64.

Calvo, M. G., Avero, P., \& Lundqvist, D. (2006). Facilitated detection of angry faces: Initial orienting and processing efficiency. Cognition and Emotion, 20, 785-811.

Carmeli, E., Patish, H., \& Coleman, R. (2003). The aging hand. The Journals of Gerontology Series A: Biological Sciences and Medical Sciences, 58(2), 146-152.

Chen, M., \& Bargh, J. A. (1999). Nonconscious approach and avoidance behavioral consequences of the automatic evaluation effect. Personality and Social Psychology Bulletin, 25, 215-224.

Culmer, P. R., Levesley, M. C., Mon-Williams, M., \& Williams, J. H. (2009). A new tool for assessing human movement: The Kinematic Assessment Tool. Journal of Neuroscience Methods, 184, 184-192.

Darwin, C. (1904). The expression of emotions in man and animals. London, UK: Murray (Original work published 1872).

Dimberg, U., \& Öhman, A. (1996). Behold the wrath: Psychophysiological responses to facial stimuli. Motivation and Emotion, 20, 149-182. doi:10.1007/BF02253869

Eastwood, J. D., Smilek, D., \& Merikle, P. M. (2001). Differential attentional guidance by unattended faces expressing positive and negative emotion. Perception \& Psychophysics, 63, 1004-1013. doi:10.3758/BF03194519

Eder, A. B., \& Rothermund, K. (2008). When do motor behaviors (mis)match affective stimuli? An evaluative coding view of 
approach and avoidance reactions. Journal of Experimental Psychology: General, 137, 262-281.

Fockenberg, D. A., Koole, S. L., Lakens, D., \& Semin, G. R. (2013). Shifting evaluation windows: Predictable forward primes with long SOAs eliminate the impact of backward primes. PLOS ONE, 8, e54739. doi:10.1371/journal.pone.0054739

Forgas, J. P. (1995). Mood and judgment: the affect intrusion model (AIM). Psychological Bulletin, 117, 39-66.

Foroni, F., \& Semin, G. R. (2009). Language that puts you in touch with your bodily feelings: The Multimodal responsiveness of affective expressions. Psychological Science, 20, 974-980.

Foroni, F., \& Semin, G. R. (2011). When does mimicry affect evaluative judgment? Emotion, 11, 687-690.

Foroni, F., \& Semin, G. R. (2012). Not all implicit measures of attitudes are created equal: Evidence from an embodiment perspective. Journal of Experimental Social Psychology, 48, 424-427.

Fox, E., Russo, R., \& Dutton, K. (2002). Attentional bias for threat: Evidence for delayed disengagement from emotional faces. Cognition and Emotion, 16, 355-379.

Gainotti, G. (2012). Unconscious processing of emotions and the right hemisphere. Neuropsychologia, 50, 205-218.

Hodsoll, S., Viding, E., \& Lavie, N. (2011). Attentional capture by irrelevant emotional distractor faces. Emotion, 11, 346-353.

Horstmann, G., Borgstedt, K., \& Heumann, M. (2006). Flanker effects with faces may depend on perceptual as well as emotional differences. Emotion, 6, 28-39. doi:10.1037/1528-3542.6.1.28

Howard, L. A., \& Tipper, S. P. (1997). Hand deviations away from visual cues: Indirect evidence for inhibition. Experimental Brain Research, $113,144-152$.

Johnson, E. J., \& Tversky, A. (1983). Affect, generalization, and the perception of risk. Journal of personality and social psychology, 45(1), 20.

Langton, S. R., Law, A. S., Burton, A. M., \& Schweinberger, S. R. (2008). Attention capture by faces. Cognition, 107, 330-342.

Milner, D. A. (1996). The visual brain in action. Oxford, UK: Oxford University Press.

Moher, J., \& Song, J.-H. (2013). Context-dependent sequential effects of target selection for action. Journal of Vision, 13(8), 10:1-13.

Nasrallah, M., Carmel, D., \& Lavie, N. (2009). "Murder she wrote": Enhanced sensitivity to negative word valence. Emotion, 9, 609-618.

Niedenthal, P. M., \& Kitayama, S. E. (1994). The heart's eye: Emotional influences in perception and attention. San Diego, CA: Academic Press.

Öhman, A. (1993). Fear and anxiety as emotional phenomena: Clinical phenomenology, evolutionary perspectives, and information processing mechanisms. In M. Lewis \& J. M. Haviland (Eds.), Handbook of emotions (pp. 511-536). New York, NY: Guilford Press.

Öhman, A., Flykt, A., \& Esteves, F. (2001). Emotion drives attention: Detecting the snake in the grass. Journal of Experimental Psychology: General, 130, 466-478. doi:10.1037/0096-3445.130.3.466

Öhman, A., \& Soares, J. J. F. (1998). Emotional conditioning to masked stimuli: Expectancies for aversive outcomes following nonrecognized fear-relevant stimuli. Journal of Experimental Psychology. General, 127, 69-82. doi:10.1037/0096-3445.127.1.69

Olszanowski, M., Pochwatko, G., Kukliński, K., Ścibor-Rylski, M., \& Ohme, R. (2008, June). Warsaw Set of Emotional Facial Expression Pictures: Validation study. Paper presented at the EAESP General Meeting, Opatija, Croatia.

Phelps, E. A., \& Anderson, A. K. (1997). Emotional memory: what does the amygdala do? Current Biology, 7, 311-314.

Rosenbaum, D. A. (1985). Motor programming: A review and scheduling theory. In H. Heuer, U. Kleinbeck, \& K.-H. Schmidt (Eds.), Motor behavior: Programming, control, and acquisition (pp. 1-33). Berlin, Germany: Springer. doi:10.1007/978-3-64269749-4 1

Sato, H., Takenaka, I., \& Kawahara, J. (2012). The effects of acute stress and perceptual load on distractor interference. Quarterly Journal of Experimental Psychology, 65, 617-623.

Schwarz, N., \& Clore, G. L. (1996). Feelings and phenomenal experience. In E. T. Higgins \& A. W. Kruglanski (Eds.), Social psychology: Handbook of basic principles (pp. 433-465). New York, NY: Guilford Press.

Simone, P. M., \& Baylis, G. C. (1997). Selective attention in a reaching task: Effect of normal aging and alzheimer's disease. Journal of Experimental Psychology: Human Perception and Performance, 23(3), 595

Song, J.-H., \& Nakayama, K. (2006). Role of focal attention on latencies and trajectories of visually guided manual pointing. Journal of Vision, 6(9), 982-995. doi:10.1167/6.9.11

Song, J.-H., \& Nakayama, K. (2009). Hidden cognitive states revealed in choice reaching tasks. Trends in Cognitive Sciences, 13, 360-366. doi:10.1016/j.tics.2009.04.009

Spoor, J. R., \& Kelly, J. R. (2004). The evolutionary significance of affect in groups: Communication and group bonding. Group Processes and Intergroup Relations, 7, 398-412. doi:10.1177/ 1368430204046145

Stephens, A., Trawley, S. L., Madigan, R., \& Groeger, J. A. (2013). Drivers display anger-congruent attention to potential traffic hazards. Applied Cognitive Psychology, 27, 178-189.

Tipper, S. P., Howard, L. A., \& Houghton, G. (1998). Action-based mechanisms of attention. Philosophical Transactions of the Royal Society, 353, 1385-1393.

Welsh, T., \& Elliott, D. (2004). Movement trajectories in the presence of a distracting stimulus: Evidence for a response activation model of selective reaching. Quarterly Journal of Experimental Psychology, 57, 1031-1057. doi:10.1080/02724980343000666

Welsh, T. N., \& Elliott, D. (2005). The effects of response priming on the planning and execution of goal-directed movements in the presence of a distracting stimulus. Acta Psychologica, 119, 123-142. doi:10. 1016/j.actpsy.2005.01.001

Zajonc, R. B. (1998). Emotions. In D. T. Gilbert, S. T. Fiske, \& G. Lindzey (Eds.), The handbook of social psychology (4th ed., pp. 591-632). Boston: McGraw-Hill. 